\title{
Selection rules for the decay of a particle into two identical massless particles of any spin
}

\author{
Seong Youl Choi $\odot^{*}$ and Jae Hoon Jeong $\odot^{\dagger}$ \\ Department of Physics and RIPC, Jeonbuk National University, Jeonju 54896, Korea
}

(Received 3 March 2021; accepted 6 April 2021; published 14 May 2021)

\begin{abstract}
The well-known Landau-Yang (LY) theorem on the decay of a neutral particle into two photons is generalized for analyzing the decay of a neutral or charged particle into two identical massless particles of any spin. Selection rules categorized by discrete parity invariance and Bose/Fermi symmetry are worked out in the helicity formulation. The general form of the Lorentz-covariant triple vertices are derived and the corresponding decay helicity amplitudes are explicitly calculated in the Jacob-Wick convention. After checking the consistency of all the analytic results obtained by two complementary approaches, we extract out the key aspects of the generalized LY theorem.
\end{abstract}

DOI: $10.1103 /$ PhysRevD.103.096013

\section{INTRODUCTION}

In the Standard Model (SM) [1-4], the photon and gluons are spin-1 massless particles. The direct observation of gravitational waves [5-7] strongly indicates the presence of spin-2 massless bosons called gravitons at the quantum level. On the other hand, it is not yet clear whether the lightest neutrino is massless or not [8] and it is a hotly debated issue whether there can exist any massless particle with its spin larger than two [9-11]. In this situation, any conceptual and/or practical studies of massless particles with arbitrary spin are still of great interest.

More than seventy years ago, Landau [12] and Yang [13] have shown that selection rules governing the decay of a particle into two photons, which are the prototype of spin-1 massless particles, can be derived from the general principle of invariance under rotation and inversion. Concisely speaking, in terms of the number $n$ of possible two-photon states for the spin $J$ and intrinsic parity $\eta$ of the decaying particle can the selection rules be summarized collectively with the compact notation $n[J]^{\eta}$ as

$n[J]^{\eta}=1[0]^{+}, \quad 2[2 k]^{+}, \quad 1[2 k+1]^{+}, \quad 1[0]^{-}, \quad 1[2 k]^{-}$,

with a positive integer $k$. One consequence of the so-called Landau-Yang (LY) theorem is that no on-shell spin-1 particle can decay into two on-shell massless photons.

\footnotetext{
sychoi@jbnu.ac.kr

jaehoonjeong229@gmail.com
}

Published by the American Physical Society under the terms of the Creative Commons Attribution 4.0 International license. Further distribution of this work must maintain attribution to the author(s) and the published article's title, journal citation, and DOI. Funded by SCOAP ${ }^{3}$.
[Therefore, both the gluon-fusion production and the twophoton decay of the resonance with mass of $125 \mathrm{GeV}$ discovered and confirmed at the LHC $[14,15]$ exclude the possibility of the spin being unity, i.e., $J \neq 1$ [16].]

In this article, we generalize the LY theorem for investigating the decay $X \rightarrow M M$ of a neutral or charged particle into two identical massless particles of any spin. We note in passing that, based on the approaches by Landau [12], the generalization of the LY theorem to the case with spin-0 and spin-1/2 massless particles has been investigated in Ref. [17] and a few partial implications of the generalized LY theorem have been worked out in Refs. [18-20]. Besides, although not studied here, we note that the LY theorem may be avoided if some of the basic assumptions for the theorem are not imposed [21-26].

First, we work out the selection rules categorized by discrete parity invariance and Bose/Fermi symmetry due to two identical bosons/fermions on the decay in the helicity formulation based on the Jacob-Wick convention [27] in Sec. II. Second, we derive the general form of the Lorentz covariant triple vertices explicitly in Sec. III and then calculate the corresponding decay helicity amplitudes in detail in Sec. IV. ${ }^{1}$ After checking the consistency of all the analytic results obtained by two complementary approaches, we summarize the key aspects of the generalized LY theorem and conclude in Sec. V. All the formulas useful for explicitly deriving the decay helicity amplitudes in the main text are listed in Appendixes A and B.

\footnotetext{
${ }^{1}$ Another convenient procedure for describing the $X M M$ triple vertex is to use a spinor formalism developed for handling massive as well as massless particles in Ref. [28].
} 


\section{SELECTION RULES IN THE HELICITY FORMALISM}

The helicity formalism [27,29] is one of the most efficient tools for discussing the two-body decay of a spin- $J$ particle $X$ into two of a massless particle $M$ of spin $s$, treating any massive and massless particles on an equal footing. For the sake of a transparent and straightforward analytic analysis, we describe the two-body decay $X \rightarrow M M$ in the $X$ rest frame $(X \mathrm{RF})$

$$
X(p, \sigma) \rightarrow M\left(k_{1}, \lambda_{1}\right)+M\left(k_{2}, \lambda_{2}\right)
$$

in terms of the momenta, $\left\{p, k_{1}, k_{2}\right\}$, and helicities, $\left\{\sigma, \lambda_{1}, \lambda_{2}\right\}$, of the particles, as depicted in Fig. 1 .

Before going into a detailed description of the general Lorentz-covariant form $X M M$ vertex in Sec. III, we study general restrictions on the decay helicity amplitude due to space inversion and Bose/Fermi symmetry for two identical massless bosons/fermions in the final state.

The helicity amplitude of the decay $X \rightarrow M M$ is decomposed in terms of the polar and azimuthal angles, $\theta$ and $\phi$, defining the direction of one massless particle in a fixed coordinate system as

$$
\begin{aligned}
\mathcal{M}_{\sigma ; \lambda_{1}, \lambda_{2}}^{X \rightarrow M M}(\theta, \phi)= & \mathcal{C}_{\lambda_{1}, \lambda_{2}} d_{\sigma, \lambda_{1}-\lambda_{2}}^{J}(\theta) e^{i\left(\sigma-\lambda_{1}+\lambda_{2}\right) \phi} \\
& \text { with } \quad\left|\lambda_{1}-\lambda_{2}\right| \leq J,
\end{aligned}
$$

with the constraint $\left|\lambda_{1}-\lambda_{2}\right| \leq J$ in the Jacob-Wick convention [27,29] (see Fig. 1 for the kinematic configuration). Here, the helicity $\sigma$ of the spin- $J$ massive particle $X$ takes one of $2 J+1$ values between $-J$ and $J$. In contrast, the helicities, $\lambda_{1,2}$, can take only two values $\pm s$ because only the maximal-magnitude helicity values are allowed for any massless particle. Therefore, there exist at most four

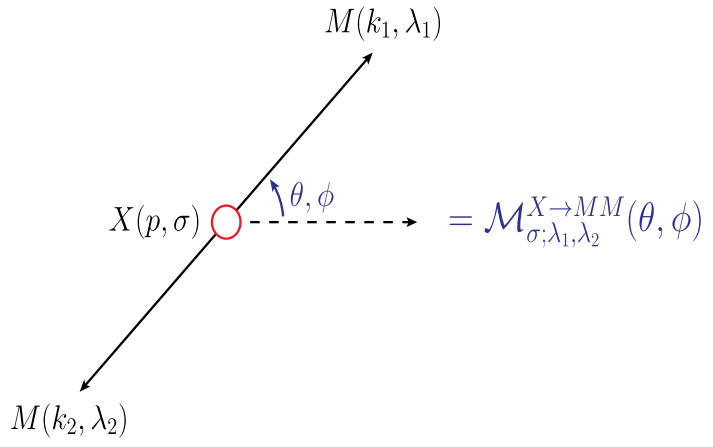

FIG. 1. Kinematic configuration for the helicity amplitude of the two-body decay $X \rightarrow M M$ of $X$ into two identical massless particles $M M$ in the $X$ rest frame (XRF). The notations, $\left\{p, k_{1,2}\right\}$ and $\left\{\sigma, \lambda_{1,2}\right\}$, are the momenta and helicities of the decaying particle $X$ and two massless particles $M M$, respectively. The polar and azimuthal angles, $\theta$ and $\phi$, are defined with respect to an appropriately chosen coordinate system. independent $\left(\lambda_{1}, \lambda_{2}\right)$ combinations $( \pm s, \pm s)$ and $( \pm s, \mp s)$, to be denoted by the shortened notations $( \pm, \pm)$ and $( \pm, \mp)$ in the following. We note that the reduced helicity amplitudes $\mathcal{C}_{\lambda_{1}, \lambda_{2}}$ do not depend on the $X$ helicity $\sigma$ due to rotational invariance and the polar-angle dependence is fully encoded in the Wigner $d$ function $d_{\sigma, \lambda_{1}-\lambda_{1}}^{J}(\theta)$ given in the convention of Rose [30].

Bose or Fermi symmetry for the two identical integer or half-integer spin particles in the final state leads to the relation for the reduced decay helicity amplitudes:

$$
\mathcal{C}_{\lambda_{1}, \lambda_{2}}=(-1)^{J} \mathcal{C}_{\lambda_{2}, \lambda_{1}} \quad \text { with } \quad\left|\lambda_{1}-\lambda_{2}\right| \leq J
$$

derived by the (anti)-symmetrization of the final state of two identical particles. If the decay process conserves parity, the reduced helicity amplitudes satisfy the spaceinversion or parity relation

$$
\mathcal{C}_{\lambda_{1}, \lambda_{2}}=\eta(-1)^{J-2 s} \mathcal{C}_{-\lambda_{1},-\lambda_{2}} \quad \text { with } \quad\left|\lambda_{1}-\lambda_{2}\right| \leq J,
$$

with $\eta$ being the intrinsic $X$ parity. Once more, we emphasize that there are only four helicity combinations $( \pm, \pm)$ and $( \pm, \mp)$ for the final-state system of two massless particles.

Remarkably, the identical-particle (ID) condition (4) and parity (PA) relation (5) enable us to straightforwardly derive the selection/exclusion rules for the two-body decay $X \rightarrow M M$ classified according to the spin $J$, the spin $s$, the $X$ intrinsic parity $\eta$ and also whether $J<2 s$ or not. The opposite-helicity amplitudes, $\mathcal{C}_{ \pm, \mp}$, vanish for $J<2 s$ and the same-helicity amplitudes, $\mathcal{C}_{ \pm, \pm}$, vanish for any odd integer $J$. Consequently, one important exclusion rule is that any decay with odd $J$ less than $2 s$ is forbidden irrespective of the $X$ parity. Specifically, the well-known rule that any spin-1 particle cannot decay into two identical massless spin-1 particles such as photons and color-neutral gluons can be guaranteed because $J=1$ is odd and $J=1<2 s=2$ for $s=1$. Moreover, for $J \geq 2 s$, the same-helicity amplitudes still vanish for any odd $J$. According to the ID condition (4) and the PA relation (5), the opposite-helicity amplitudes survive only when $\eta(-1)^{2 s}=+1$ with the relation $\mathcal{C}_{+,-}=(-1)^{J} \mathcal{C}_{-,+}$.

Based on the above observations, a few interesting selection rules can be extracted out as follows:

(i) No odd- $J$ particle can decay into two identical spin0 massless scalar bosons $(s=0)$.

(ii) The allowed decay of a spin-1 massive particle into two identical massless particles is only into two identical spin-1/2 massless fermions, when the intrinsic parity $\eta$ of the decaying particle is odd.

(iii) Any even-parity and odd-spin particle cannot decay into two identical massless fermions.

(iv) Any odd-parity and odd-spin particle cannot decay into two identical massless bosons.

Table I summarizes all the selection/exclusion rules on the special decay $X \rightarrow M M$. We note that the case with $s=0$ is 
TABLE I. Selection rules for the decay $X \rightarrow M M$. The values, $J$ and $s$, are the spins of the massive $X$ and massless $M$ particles, respectively, and the signature \pm denotes the sign of the product $\zeta \equiv \eta(-1)^{J-2 s}$ of the $X$ intrinsic parity $\eta$ and the factor $(-1)^{J-2 s}$. The mark [forbidden] means that the corresponding term is absent when $s=0$.

\begin{tabular}{lccc}
\hline \hline $\boldsymbol{J}$ & $J<2 s$ & $J \geq 2 s[s=0]$ & \\
\hline odd & $\begin{array}{c}\zeta= \pm \\
\text { forbidden }\end{array}$ & $\begin{array}{c}\zeta=+ \\
\text { forbidden }\end{array}$ & $\begin{array}{c}\mathcal{C}_{+,-}=- \\
=-\mathcal{C}_{-,+}[\text {forbidden] }\end{array}$ \\
even & $\begin{array}{c}\zeta= \pm \\
\mathcal{C}_{+,+}= \pm \mathcal{C}_{-,-}\end{array}$ & $\begin{array}{c}\zeta=- \\
\mathcal{C}_{+,-}=\mathcal{C}_{-,+}\left[\text {forbidden], } \mathcal{C}_{+,+}=\mathcal{C}_{-,-}\right.\end{array}$ & $\mathcal{C}_{+,+}=-\mathcal{C}_{-,-}[$forbidden] \\
\hline \hline
\end{tabular}

more restricted than the case with nonzero spin $s$, as marked with the comment [forbidden].

Interestingly, as pointed out in Ref. [28], the general selection rules enable us to reinterpret the Weinberg-Witten (WW) theorem [9]. Since conserved currents and stress tensors measure the charge and momentum on single particle states respectively, let us consider the decay of a massive neutral particle into two identical massless particles with opposite helicities, i.e., $\lambda_{1}=-\lambda_{2}= \pm$, because two momenta $k_{1}$ and $k_{2}$ are out going and so $\lambda_{1}=-\lambda_{2}$ in order for $k_{1}$ and $k_{2}$ to represent the same particle. In this case, as can be checked with Table I, the inequality $s \leq J / 2$ must be satisfied, i.e., $s \leq 1 / 2$ for $J=1$ and $s \leq 1$ for $J=2$. That is to say, massless particles with spin $s>1 / 2$ cannot couple to a Lorentz covariant conserved current corresponding to $J=1$ and those with spin $s>1$ cannot couple to a conserved energy-momentum stress tensor corresponding to $J=2$.

In the next section, we show how the selection rules derived in the helicity formalism are reflected and encoded in the Lorentz-covariant form of the triple $X M M$ vertex in the integer and half-integer $s$ cases separately.

\section{GENERAL LORENTZ-COVARIANT TRIPLE VERTEX TENSORS}

Generically, the decay amplitude of one on-shell particle $X$ of mass $m$ and integer spin $J$ into two of a massless particle $M$ of spin $s$ can be written in terms of the triple vertex tensor $\Gamma$ as (See Fig. 2 for its diagrammatic description)

$\mathcal{M}_{\sigma ; \lambda_{1}, \lambda_{2}}^{X \rightarrow M M}=\bar{u}_{1}^{\alpha}\left(k_{1}, \lambda_{1}\right) \Gamma_{\alpha, \beta}^{\mu}(p, k) v_{2}^{\beta}\left(k_{2}, \lambda_{2}\right) \epsilon_{\mu}(p, \sigma)$,

with $\lambda_{1,2}= \pm s= \pm$ for the two-body decay $X \rightarrow M M$, where $p$ and $\sigma$ are the momentum and helicity of the particle $X$, and $k_{1,2}$ and $\lambda_{1,2}$ are the momenta and helicities of two massless particles, respectively. Here, $p=k_{1}+k_{2}$ and $k=k_{1}-k_{2}$, are symmetric and antisymmetric under the interchange of two momenta, $k_{1}$ and $k_{2}$. The index $\mu$ stands collectively for $\mu=\mu_{1} \cdots \mu_{J}$. If $s=n$ is an integer, then the wave tensors $\bar{u}_{1}^{\alpha}\left(k_{1}, \lambda_{1}\right)$ and $v_{2}^{\beta}\left(k_{2}, \lambda_{2}\right)$ with $\lambda_{1,2}= \pm s$ are given by

$$
\begin{aligned}
& \bar{u}_{1}^{\alpha}\left(k_{1}, \pm s\right)=\epsilon_{1}^{* \alpha_{1} \cdots \alpha_{n}}\left(k_{1}, \pm n\right), \\
& v_{2}^{\beta}\left(k_{2}, \pm s\right)=\epsilon_{2}^{* \beta_{1} \cdots \beta_{n}}\left(k_{2}, \pm n\right),
\end{aligned}
$$

and if $s=n+1 / 2$ is a half-integer, the wave tensors are given by

$$
\begin{aligned}
& \bar{u}_{1}^{\alpha}\left(k_{1}, \pm s\right)=\epsilon_{1}^{* \alpha_{1} \cdots \alpha_{n}}\left(k_{1}, \pm n\right) \bar{u}_{1}\left(k_{1}, \pm\right), \\
& v_{2}^{\beta}\left(k_{2}, \pm s\right)=\epsilon_{2}^{* \beta_{1} \cdots \beta_{n}}\left(k_{2}, \pm n\right) v_{2}\left(k_{2}, \pm\right),
\end{aligned}
$$

where $\bar{u}_{1}\left(k_{1}, \pm\right)=u_{1}^{\dagger}\left(k_{1}, \pm\right) \gamma^{0}$ and the $u_{1}\left(k_{1}, \pm\right)$ and $v_{2}\left(k_{2}, \pm\right)$ are the spin-1/2 particle $u_{1}$ and antiparticle $v_{2}$ spinors. (See Appendix A for their detailed expressions.)

An on-shell boson of integer spin $J$, nonzero mass $m$, momentum $p$ and helicity $\sigma$ is defined by a rank- $J$ tensor $\epsilon_{\mu_{1} \cdots \mu_{J}}(p, \sigma)[31-33]$ that is completely symmetric, traceless and divergence-free

$$
\begin{gathered}
\varepsilon^{\alpha \beta \mu_{i} \mu_{j}} \epsilon_{\mu_{1} \cdots \mu_{i} \cdots \mu_{j} \cdots \mu_{J}}(p, \sigma)=0, \\
g^{\mu_{i} \mu_{j}} \epsilon_{\mu_{1} \cdots \mu_{i} \cdots \mu_{j} \cdots \mu_{J}}(p, \sigma)=0,
\end{gathered}
$$

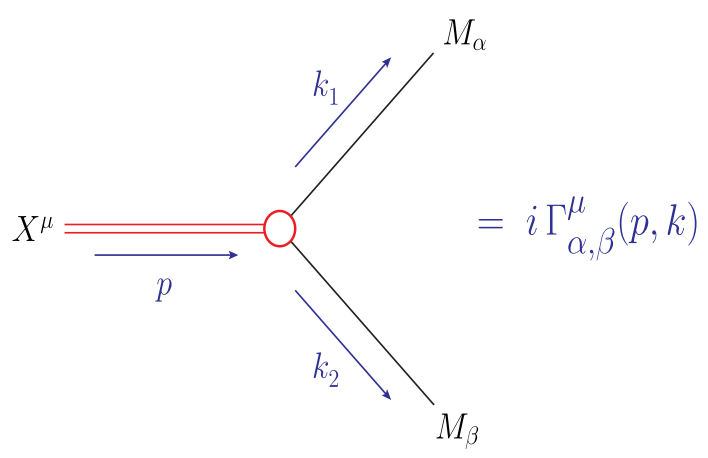

FIG. 2. Feynman rules for the general $X M M$ triple vertex of a spin- $J$ particle $X$ and two identical spin-s massless particles $M M$. The indices, $\mu, \alpha$ and $\beta$, stand for the sequences of $\mu=$ $\mu_{1} \cdots \mu_{J}, \alpha_{1} \cdots \alpha_{n}$ and $\beta_{1} \cdots \beta_{n}$, collectively. The integer $n$ is $s$ for a boson $X$ and $s-1 / 2$ for a fermion $X$ for the $X$ spin $s$. The symmetric and antisymmetric momentum combinations, $p=$ $k_{1}+k_{2}$ and $k=k_{1}-k_{2}$, are introduced for systematic classifications of the triple vertex tensor. 


$$
p^{\mu_{i}} \epsilon_{\mu_{1} \cdots \mu_{i} \cdots \mu_{J}}(p, \sigma)=0,
$$

and it satisfies the on-shell wave equation $\left(p^{2}-m^{2}\right) \epsilon_{\mu_{1} \cdots \mu_{J}}$ $(p, \sigma)=0$ for any helicity value of $\sigma$ taking an integer between $-J$ and $J$. The wave tensor can be expressed explicitly by a linear combination of $J$ products of spin-1 wave vectors with appropriate Clebsch-Gordon coefficients.

\section{A. Massless particles of integer spin}

The wave tensor of a massless particle of integer spin $s$ is given simply by a product of $s$ spin-1 wave vectors, each of which carries the same helicity of \pm 1 , as

$$
\epsilon^{\alpha_{1} \cdots \alpha_{s}}(k, \pm s)=\epsilon^{\alpha_{1}}(k, \pm) \cdots \epsilon^{\alpha_{s}}(k, \pm),
$$

where, for notational convenience, the notation \pm is used for \pm 1 . The wave tensor (14) also is completely symmetric, traceless and divergence-free and it satisfies the wave equation $k^{2} \epsilon^{\alpha_{1} \cdots \alpha_{s}}(k, \lambda)=0$ with $k^{2}=0$ automatically.

To begin with, let us consider as a special case the decay $X \rightarrow \gamma \gamma$ of a massive integer-spin boson $X$ into two massless spin-1 photons, originally investigated by Landau [12] and Yang [13]. Imposing the on-shell conditions valid for two spin-1 massless photons ${ }^{2}$

$$
k_{i} \cdot \epsilon_{i}\left(k_{i}, \lambda_{i}\right)=0 \quad \text { and } \quad k_{i}^{2}=0 \quad[i=1,2] \text {, }
$$

and performing the Bose symmetrization of two identical $\gamma$ states in the final state, we can write the general $X \gamma \gamma$ vertex in a greatly-simplified form in terms of four independent parameters, $x_{\gamma}^{ \pm}$and $y_{\gamma}^{ \pm}$, as [34]

$$
\begin{aligned}
\Gamma_{\mu ; \alpha, \beta}^{X \rightarrow \gamma \gamma}(p, k)= & \eta_{+} x_{\gamma}^{+} g_{\perp \alpha \beta} k_{\mu_{1}} \cdots k_{\mu_{J}} / m^{J}+\eta_{+} x_{\gamma}^{-} \imath\langle\alpha \beta p k\rangle k_{\mu_{1}} \cdots k_{\mu_{J}} / m^{J+2} \\
& +\eta_{+} y_{\gamma}^{+}\left(g_{\perp \alpha \mu_{1}} g_{\perp \beta \mu_{2}}+g_{\perp \beta \mu_{1}} g_{\perp \alpha \mu_{2}}-g_{\perp \alpha \beta} k_{\mu_{1}} k_{\mu_{2}} / m^{2}\right) k_{\mu_{3}} \cdots k_{\mu_{J}} / m^{J-2} \\
& +\eta_{-} y_{\gamma}^{-} \imath\left(g_{\perp \alpha \mu_{1}}\left\langle\beta \mu_{2} p k\right\rangle+g_{\perp \beta \mu_{1}}\left\langle\alpha \mu_{2} p k\right\rangle\right) k_{\mu_{3}} \cdots k_{\mu_{J}} / m^{J}
\end{aligned}
$$

satisfying the orthogonality conditions, $k_{1}^{\alpha} \Gamma_{\mu ; \alpha, \beta}^{X \rightarrow \gamma \gamma}(p, k)=$ $k_{2}^{\beta} \Gamma_{\mu ; \alpha, \beta}^{X \rightarrow \gamma \gamma}(p, k)=0$, with $\mu$ denoting collectively $\mu_{1} \cdots \mu_{J}$, the projection factors, $\eta_{ \pm}=\left[1 \pm(-1)^{J}\right] / 2$, and two momentum combinations, $p=k_{1}+k_{2}$ and $k=k_{1}-k_{2}$, which are symmetric and antisymmetric under the interchange of two massless particles, i.e., $k_{1} \leftrightarrow k_{2}$ and $\alpha \leftrightarrow \beta$, respectively. The antisymmetric tensor $\langle\alpha \beta p k\rangle=\varepsilon_{\alpha \beta \rho \sigma} p^{\rho} k^{\sigma}$ in terms of the totally antisymmetric Levi-Civita tensor with the sign convention $\varepsilon_{0123}=+1$. For the sake of notation, the following orthogonal tensors are introduced,

$$
\begin{aligned}
g_{\perp \alpha \beta} & =g_{\alpha \beta}-2 k_{2 \alpha} k_{1 \beta} / m^{2}, \\
g_{\perp \alpha \mu_{i}} & =g_{\alpha \mu_{i}}-2 p_{\alpha} k_{1 \mu_{i}} / m^{2}, \\
g_{\perp \beta \mu_{i}} & =g_{\beta \mu_{i}}-2 p_{\beta} k_{2 \mu_{i}} / m^{2},
\end{aligned}
$$

with $i=1, \ldots, J$. The totally symmetric wave tensor $\epsilon^{\mu_{1} \cdots \mu_{J}}(p, \sigma)$ to be coupled to the triple vertex (16) guarantees the automatic symmetrization of all the $x_{\gamma}^{ \pm}$and $y_{\gamma}^{ \pm}$ terms under any $\mu$-index permutations. It is straightforward to derive the following selection rules from the expression of the $X \gamma \gamma$ vertex in Eq. (16),

(i) The $Y_{1,2}^{+}$terms survive for non-negative even integers, $J=0,2,4$, and so on.

\footnotetext{
${ }^{2}$ While the on-shell conditions (15) are maintained, the wave vector $\epsilon_{i}^{\mu}$ of a spin-1 massless particle can be adjusted by adding any term proportional to $k_{i}^{\mu}$, guaranteeing that its spatial part orthogonal to $\vec{k}_{i}$ remains as two physical degrees of freedom.
}

(ii) The $Y_{3}^{+}$term survives for positive even integers, $J=2,4$, and so on, satisfying $J \geq 2$.

(iii) The $Y_{1}^{-}$term survives for positive odd integers, $J=3,5$, and so on, satisfying $J>2$.

One immediate consequence is that any massive on-shell spin-1 particle with $J=1$ cannot decay into two on-shell identical massless spin-1 particles such as photons, because the three $x_{\gamma}^{ \pm}$and $y_{\gamma}^{+}$terms vanish with $\eta_{+}=0$ for $J=1$ and the $y_{\gamma}^{-}$term surviving only for odd $J$ contribute to the vertex only for $J \geq 3$.

For a massless boson $M=b$ of an arbitrary integer spin $s$, the general Lorentz-covariant form of the $X b b$ vertex can be written compactly by introducing two scalar-type operators and two tensor-type operators as

$$
\begin{gathered}
S_{\alpha_{i} \beta_{i}}^{+}=g_{\perp \alpha_{i} \beta_{i}}, \\
S_{\alpha_{i} \beta_{i}}^{-}=\imath\left\langle\alpha_{i} \beta_{i} p k\right\rangle / m^{2} \\
T_{\alpha_{i} \beta_{i}, \mu_{2 i-1} \mu_{2 j}}^{+}=g_{\perp \alpha_{i} \mu_{2 i-1}} g_{\perp \beta_{i} \mu_{2 i}}+g_{\perp \beta_{i} \mu_{2 i-1}} g_{\perp \alpha_{i} \mu_{2 i}} \\
-g_{\perp \alpha_{i} \beta_{i}} k_{\mu_{2 i-1}} k_{\mu_{2 j}} / m^{2} \\
T_{\alpha_{i} \beta_{i}, \mu_{2 i-1} \mu_{2 i}}^{-}=l\left(g_{\perp \alpha_{i} \mu_{2 i-1}}\left\langle\beta_{i} \mu_{2 i} p k\right\rangle+g_{\perp \beta_{i} \mu_{2 i-1}}\left\langle\alpha_{i} \mu_{2 i} p k\right\rangle\right) / m^{2}
\end{gathered}
$$

with $i=1, \ldots, s$. The general form of the vertex tensor is then cast into a compact form as 


$$
\begin{aligned}
\Gamma_{\mu ; \alpha, \beta}^{X \rightarrow b b}(p, k)= & \eta_{+}\left(x_{b}^{+} S_{\alpha_{1} \beta_{1}}^{+}+x_{b}^{-} S_{\alpha_{1} \beta_{1}}^{-}\right) S_{\alpha_{2} \beta_{2}}^{+} \cdots S_{\alpha_{s} \beta_{s}}^{+} k_{\mu_{1}} \cdots k_{\mu_{J}} / m^{J} \\
& +\eta_{+} \theta(J-2 s) y_{b}^{+} T_{\alpha_{1} \beta_{1}, \mu_{1} \mu_{2}}^{+} T_{\alpha_{2} \beta_{2}, \mu_{3} \mu_{4}}^{+} \cdots T_{\alpha_{s} \beta_{s}, \mu_{2 s-1} \mu_{2 s}}^{+} k_{\mu_{2 s+1}} \cdots k_{\mu_{J}} / m^{J-2 s} \\
& +\eta_{-} \theta(J-2 s) y_{b}^{-} T_{\alpha_{1} \beta_{1}, \mu_{1} \mu_{2}}^{-} T_{\alpha_{2} \beta_{2}, \mu_{3} \mu_{4}}^{+} \cdots T_{\alpha_{s} \beta_{s}, \mu_{2 s-1} \mu_{2 s}}^{+} k_{\mu_{2 s+1}} \cdots k_{\mu_{J}} / m^{J-2 s}
\end{aligned}
$$

in terms of four independent parameters, $x_{b}^{ \pm}$and $y_{b}^{ \pm}$, with $\eta_{ \pm}=\left[1 \pm(-1)^{J}\right] / 2$ and the step function $\theta(J-2 s)=1$ and 0 for $J \geq 2 s$ and $J<2 s$. Here, the indices, $\mu, \alpha$, and $\beta$, stand collectively for $\mu=\mu_{1} \cdots \mu_{J}, \alpha=\alpha_{1} \cdots \alpha_{s}$, and $\beta=\beta_{1} \cdots \beta_{s}$. One immediate consequence is that the case with odd $J$ less than $2 s$ is forbidden irrespective of the $X$ intrinsic parity $\eta$, which is consistent with the corresponding selection rule listed in Table I. Specifically, any spin-1 particle cannot decay into two identical spin-1 massless particles with $s=1$.

\section{B. Massless particles of half-integer spin}

An on-shell massless particle or antiparticle of a halfinteger spin $s=n+1 / 2(n=0,1,2, \ldots)$ and momentum $k$ may be described by a product of a $u$ or $v$ spinor and $n$ spin-1 wave vectors as

$$
\begin{gathered}
u^{\alpha}(k, \pm s)=\epsilon^{\alpha_{1}}(k, \pm) \cdots \epsilon^{\alpha_{n}}(k, \pm) u(k, \pm), \\
v^{\alpha}(k, \pm s)=\epsilon^{* \alpha_{1}}(k, \pm) \cdots \epsilon^{* \alpha_{n}}(k, \pm) v(k, \pm),
\end{gathered}
$$

that are traceless, symmetric and divergence-free in the indices $\alpha=\alpha_{1} \cdots \alpha_{n}$ and the $u$ and $v$ spinor tensors satisfy

$$
\begin{gathered}
\gamma_{\alpha_{i}} u^{\alpha_{1} \cdots \alpha_{i} \cdots \alpha_{n}}(k, \pm s)=\gamma_{\alpha_{i}} v^{\alpha_{1} \cdots \alpha_{i} \cdots \alpha_{n}}(k, \pm s)=0, \\
\not k u^{\alpha_{1} \cdots \alpha_{i} \cdots \alpha_{n}}(k, \pm s)=\not k v^{\alpha_{1} \cdots \alpha_{i} \cdots \alpha_{n}}(k, \pm s)=0,
\end{gathered}
$$

with $\not k=k_{\mu} \gamma^{\mu}$. We adopt the chiral representation for the Dirac gamma matrices $\gamma^{\mu}(\mu=0,1,2,3)$, whose expressions are listed in Appendix B.

Interchanging two identical massless fermions, i.e., taking the opposite fermion flow line $[35,36]$, we can rewrite the helicity amplitude of the decay $X \rightarrow f f$ with a massless fermion $M=f$ as

$$
\begin{aligned}
\tilde{\mathcal{M}}_{\sigma ; \lambda_{1}, \lambda_{2}}^{X \rightarrow f f} & =\bar{u}_{2}^{\beta}\left(k_{2}, \lambda_{2}\right) \Gamma_{\beta, \alpha}^{\mu}(p,-k) v_{1}^{\alpha}\left(k_{1}, \lambda_{1}\right) \epsilon_{\mu}(p, \sigma) \\
& =v_{1}^{T \alpha}\left(k_{1}, \lambda_{1}\right) \Gamma_{\beta, \alpha}^{\mu T}(p,-k) \bar{u}_{2}^{T \beta}\left(k_{2}, \lambda_{2}\right) \epsilon_{\mu}(p, \sigma),
\end{aligned}
$$

with the superscript $T$ denoting the transpose of the matrix. Introducing the charge-conjugation operator $C$ satisfying $C^{\dagger}=C^{-1}$ and $C^{T}=-C$ relating the $v$ spinor to the $u$ spinor as

$$
v^{\alpha}(k, \lambda)=C \bar{u}^{T \alpha}(k, \lambda)
$$

with $\bar{u}=u^{\dagger} \gamma^{0}$, we can rewrite the amplitude as

$\tilde{\mathcal{M}}_{\sigma ; \lambda_{1}, \lambda_{2}}^{X \rightarrow f f}=-\bar{u}_{1}^{\beta}\left(k_{1}, \lambda_{1}\right) C \Gamma_{\beta, \alpha}^{\mu T}(p,-k) C^{-1} v_{2}^{\alpha}\left(k_{2}, \lambda_{2}\right) \epsilon_{\mu}(p, \sigma)$.

Since Fermi statistics requires $\tilde{\mathcal{M}}=-\mathcal{M}$, the triple vertex tensor must satisfy the relation

$$
C \Gamma_{\beta, \alpha}^{\mu T}(p,-k) C^{-1}=\Gamma_{\alpha, \beta}^{\mu}(p, k),
$$

which enables us to classify all the allowed terms systematically [37-39].

The basic relation for the charge-conjugation invariance of the Dirac equation is $C \gamma_{\mu}^{T} C^{-1}=-\gamma_{\mu}$ with a unitary matrix $C$. Repeatedly using the basic relation, we can derive

$$
\begin{aligned}
& \Gamma^{c} \equiv C \Gamma^{T} C^{-1}=\epsilon_{C} \Gamma \text { with } \\
& \epsilon_{C}=\left\{\begin{array}{lll}
+1 & \text { for } \quad \Gamma=1, \gamma_{5}, \gamma_{\mu} \gamma_{5} \\
-1 & \text { for } & \Gamma=\gamma_{\mu}
\end{array}\right.
\end{aligned}
$$

There are no further independent terms as any other operator can be replaced by a linear combination of 1 , $\gamma_{5}, \gamma_{\mu}$, and $\gamma_{\mu} \gamma_{5}$ by use of the so-called Gordon identities, when coupled to the $u$ and $v$ spinors.

Because of the conditions (25) and (26), the vector structure $\gamma^{\mu}$ can be contracted only with the wave vector $\epsilon^{\mu}(p, \sigma)$ of the decaying particle $X$ with the helicity $\sigma= \pm 1,0= \pm, 0$. Effectively we can make the following replacements

$$
\begin{aligned}
S_{\alpha \beta}^{-}\left[1, \gamma_{5}\right] & \leftrightarrow-S_{\alpha \beta}^{+}\left[\gamma_{5}, 1\right], \\
\gamma_{\mu_{1}}\left[1, \gamma_{5}\right] T_{\alpha \beta, \mu_{2} \mu_{3}}^{-} & \leftrightarrow \gamma_{\mu_{1}}\left[\gamma_{5}, 1\right] T_{\alpha \beta, \mu_{2} \mu_{3}}^{+},
\end{aligned}
$$

because two corresponding terms in each equation give rise to the same helicity amplitudes as shown explicitly in Appendix B. Then, for a massless fermion $M=f$, the general form of the $X f f$ triple vertex tensor can be written as 


$$
\begin{aligned}
\Gamma_{\mu ; \alpha, \beta}^{X \rightarrow f f}(p, k)= & \eta_{+}\left(x_{f}^{+}+x_{f}^{-} \gamma_{5}\right) S_{\alpha_{1} \beta_{1}}^{+} \cdots S_{\alpha_{n} \beta_{n}}^{+} k_{\mu_{1}} \cdots k_{\mu_{J}} / m^{J} \\
& +\eta_{+} \theta(J-2 s) y_{f}^{+} \gamma_{\mu_{1}} T_{\alpha_{1} \beta_{1}, \mu_{2} \mu_{3}} \cdots T_{\alpha_{n} \beta_{n}, \mu_{2 n} \mu_{2 n+1}}^{+} k_{\mu_{2 n+2}} \cdots k_{\mu_{J}} / m^{J-2 s-1} \\
& +\eta_{-} \theta(J-2 s) y_{f}^{-} \gamma_{\mu_{1}} \gamma_{5} T_{\alpha_{1} \beta_{1}, \mu_{2} \mu_{3}} \cdots T_{\alpha_{n} \beta_{n}, \mu_{2 n} \mu_{2 n+1}}^{+} k_{\mu_{2 n+2}} \cdots k_{\mu_{J}} / m^{J-2 s-1},
\end{aligned}
$$

in terms of four independent parameters, $x_{f}^{ \pm}$and $y_{f}^{ \pm}$, with $n=s-1 / 2$. The scalar part proportional to $g_{\perp \alpha_{i} \beta_{i}} k_{\mu_{2 i}} k_{\mu_{2 i+1}}$ of $T_{\alpha_{i} \beta_{i}, \mu_{2 i} \mu_{2 i+1}}^{+}$in Eq. (20) can be discarded because its contribution vanishes in the opposite helicity case with $\lambda_{1}=-\lambda_{2}$ enforced by the helicity-preserving (axial-)vector currents. It is straightforward to check that the expression (34) satisfies the Fermi symmetry condition (30) for two identical fermions. One nontrivial observation is that a spin-1 particle can decay only into two identical spin- $1 / 2$ particles [39] through an axial-vector $\gamma_{\mu} \gamma_{5}$ current as can be checked with the last expression in Eq. (34).

\section{EXPLICIT FORM OF REDUCED HELICITY AMPLITUDES}

Employing all the analytic results for the scalar, vector, and tensor currents listed in Appendix $\mathrm{B}$ enables us to explicitly calculate all the reduced helicity amplitudes $\mathcal{C}_{\lambda_{1}, \lambda_{2}}$ defined in Eq. (3) both in the integer-spin and half-integerspin cases for the massless particle.

In the case with a massless integer-spin s boson $M=b$, the reduced helicity amplitudes for the process $X \rightarrow b b$ with the same helicities $( \pm, \pm)$ read

$$
\mathcal{C}_{ \pm, \pm}=\frac{2^{J / 2} J !}{\sqrt{(2 J) !}}\left(x_{b}^{+} \pm x_{b}^{-}\right),
$$

surviving only for even-integer $J$. If parity is preserved, the term, $x_{b}^{+}$or $x_{b}^{-}$, can survive for the even or odd $X$ intrinsic parity $\eta= \pm$, respectively. On the other hand, the reduced helicity amplitudes with the opposite helicities $( \pm, \mp)$, which survive only when $J \geq 2 s$, read

$$
\mathcal{C}_{ \pm, \mp}=2^{J / 2} \sqrt{\frac{(J+2 s) !(J-2 s) !}{(2 J) !}} y_{b}^{+},
$$

for even-integer spin $J \geq 2 s$ and even $X$ intrinsic parity $\eta=+$, and

$$
\mathcal{C}_{ \pm, \mp}=\mp 2^{J / 2} \sqrt{\frac{(J+2 s) !(J-2 s) !}{(2 J) !} y_{b}^{-}},
$$

for odd-integer spin $J \geq 2 s$ and even $X$ intrinsic parity $\eta=+$.

In the case with a massless fermion $M=f$ with a half-integer spin $s$, the reduced helicity amplitudes for the process $X \rightarrow f f$ with the same helicities $( \pm, \pm)$ read

$$
\mathcal{C}_{ \pm, \pm}=\frac{2^{J / 2} J !}{\sqrt{(2 J) !}}\left(x_{f}^{+} \mp x_{f}^{-}\right)
$$

surviving only for even-integer $J$. If parity is preserved, the $x_{f}^{+}$and $x_{f}^{-}$terms can survive for the odd and even $X$ intrinsic parity $\eta=\mp$, respectively. On the other hand, the reduced helicity amplitudes, which survive only when $J \geq 2 s$, read

$$
\mathcal{C}_{ \pm, \mp}=(-1)^{2 s} 2^{J / 2} \sqrt{\frac{(J+2 s) !(J-2 s) !}{(2 J) !}} y_{f}^{+},
$$

for even-integer spin $J \geq 2 s$ and odd $X$ intrinsic parity $\eta=-$, and

$$
\mathcal{C}_{ \pm, \mp}=\mp(-1)^{2 s} 2^{J / 2} \sqrt{\frac{(J+2 s) !(J-2 s) !}{(2 J) !}} y_{f}^{-},
$$

for odd-integer spin $J \geq 2 s$ and odd $X$ intrinsic parity $\eta=-$.

\section{CONCLUSIONS}

We have generalized the well-known Landau-Yang (LY) theorem on the decay of a neutral particle into two photons for analyzing the two-body decay of a neutral or charged particle into two identical massless particles of any integer or half-integer spin. After having worked out the selection rules classified by discrete parity invariance and Bose/Fermi symmetry in the helicity formulation, we have derived the general form of the Lorentz covariant triple vertices and calculated the helicity amplitudes explicitly. After checking the consistency of all the analytic results obtained from two complementary approaches, we have drawn out the key aspects of the generalized LY theorem including the reinterpretation of the WW theorem.

Introducing a compact notation $n[J, s]^{\zeta}$ consisting of the number $n$ of independent terms, the $X$ and $M$ spins, $J$ and $s$, and a reduced parity $\zeta=\eta(-1)^{J-2 s}$ with the $X$ intrinsic parity $\eta$, we can summarize the selection rules on the decay of a massive particle into an identical pair of massless particles of arbitrary spin: 


$$
\begin{aligned}
n[J, s]^{\zeta}=1[0,0]^{+}, & 1[0, s>0]^{ \pm}, \quad 1[2 k-1,0<s \leq k-1 / 2]^{-}, \\
1[2 k, 0]^{+}, & 2[2 k, 0<s \leq k]^{+}, \quad 1[2 k, 0<s \leq k]^{-}, \quad 1[2 k, s>k]^{ \pm},
\end{aligned}
$$

with $k=1,2, \ldots$ In the special case of $s=1$, the selection rules reduce to those called the LY theorem described in Eq. (1). The massless particle for the decay of a spin-1 particle into its identical pair must have a half-integer spin $s=1 / 2$. If parity is preserved, no odd-spin and odd-parity (even-parity) particle can decay into an identical pair of massless bosons (fermions).

As natural extension of the present work, the decays of an arbitrary integer-spin particle into two identical massive particles [39] or two distinguishable charge self-conjugate particles of any spin are presently under study and the results will be reported separately. Before closing, we emphasize that the selection rules obtained in this article can be applied without any modifications, for example, to the decay of a doubly charged massive particle into two identical singly charged massless particles, where the charge can be of any type as well as of a typical electric charge type.

\section{ACKNOWLEDGMENTS}

The work was in part by the Basic Science Research Program of Ministry of Education through National Research Foundation of Korea (Grant No. NRF2016R1D1A3B01010529) and in part by the CERNKorea theory collaboration. A correspondence with Kentarou Mawatari concerning the covariant formulation of the Landau-Yang theorem is acknowledged.

\section{APPENDIX A: WAVE VECTORS AND SPINORS IN THE JACOB-WICK CONVENTION}

In Appendix A, we show the explicit expressions for the wave vectors and spinors of a massive particle $X$ and two massless particles in the $X \mathrm{RF}$ with the kinematic configuration as shown in Fig. 1. The Jacob-Wick convention of Ref. [27] is chosen for the vectors and spinors. In terms of the polar and azimuthal angles, $\theta$ and $\phi$, the three momenta, $p=k_{1}+k_{2}$ and $k_{1,2}$, and the combination $k=k_{1}-k_{2}$ read

$$
\begin{gathered}
p=m(1,0,0,0), \\
k_{1}=\frac{m}{2}(1, \sin \theta \cos \phi, \sin \theta \sin \phi, \cos \theta), \\
k_{2}=\frac{m}{2}(1,-\sin \theta \cos \phi,-\sin \theta \sin \phi,-\cos \theta), \\
k=m(0, \sin \theta \cos \phi, \sin \theta \sin \phi, \cos \theta) .
\end{gathered}
$$

For the sake of notation, we introduce three unit vectors

$$
\begin{gathered}
\hat{k}=(\sin \theta \cos \phi, \sin \theta \sin \phi, \cos \theta)=\vec{k} / m, \\
\hat{\theta}=(\cos \theta \cos \phi, \cos \theta \sin \phi,-\sin \theta), \\
\hat{\phi}=(-\sin \phi, \cos \phi, 0),
\end{gathered}
$$

which are mutually orthonormal, i.e., $\hat{k} \cdot \hat{\theta}=\hat{\theta} \cdot \hat{\phi}=\hat{\phi}$. $\hat{k}=0$ and $\hat{k} \cdot \hat{k}=\hat{\theta} \cdot \hat{\theta}=\hat{\phi} \cdot \hat{\phi}=1$.

The wave vectors for the particle with momentum $p$ and two massless particles with momenta $k_{1,2}$ are given by

$$
\begin{gathered}
\epsilon(p, \pm)=\frac{1}{\sqrt{2}}(0, \mp 1,-l, 0), \\
\epsilon(p, 0)=(0,0,0,1),
\end{gathered}
$$

$$
\begin{aligned}
\epsilon_{1}\left(k_{1}, \pm\right)= & \frac{1}{\sqrt{2}} e^{ \pm \imath \phi}(0, \mp \cos \theta \cos \phi+\imath \sin \phi, \\
& \mp \cos \theta \sin \phi-\imath \cos \phi, \pm \sin \theta) \\
= & \frac{1}{\sqrt{2}} e^{ \pm \imath \phi}(0, \mp \hat{\theta}-\imath \hat{\phi}), \\
\epsilon_{2}\left(k_{2}, \pm\right)= & \epsilon_{1}\left(k_{1}, \mp\right)=-\epsilon_{1}^{*}\left(k_{1}, \pm\right)=-\epsilon_{2}^{*}\left(k_{2}, \mp\right),
\end{aligned}
$$

where the last relation between two wave vectors is satisfied in the Jacob-Wick convention.

The spin-1/2 $u$ and $v$ 4-component spinors of the massless particles with momenta $k_{1,2}$ are given in the Jacob-Wick convention by

$$
\begin{aligned}
& u_{1}\left(k_{1},+\right)=-v_{1}\left(k_{1},-\right)=\sqrt{m}\left(\begin{array}{c}
0 \\
\chi_{+}(\hat{k})
\end{array}\right), \\
& u_{1}\left(k_{1},-\right)=-v_{1}\left(k_{1},+\right)=\sqrt{m}\left(\begin{array}{c}
\chi_{-}(\hat{k}) \\
0
\end{array}\right), \\
& u_{2}\left(k_{2},+\right)=v_{2}\left(k_{2},-\right)=\sqrt{m}\left(\begin{array}{c}
0 \\
\chi_{-}(\hat{k})
\end{array}\right), \\
& u_{2}\left(k_{2},-\right)=v_{2}\left(k_{2},+\right)=\sqrt{m}\left(\begin{array}{c}
\chi_{+}(\hat{k}) \\
0
\end{array}\right),
\end{aligned}
$$

satisfying the relations $u_{2}\left(k_{2}, \pm\right)=\gamma^{0} u_{1}\left(k_{1}, \mp\right)$ and $v_{2}\left(k_{2}, \pm\right)=-\gamma^{0} v_{1}\left(k_{1}, \mp\right)$ with the expression of $\gamma^{0}$ given 
in Appendix $\mathrm{B}$, where the 2-component spinors $\chi_{ \pm}(\hat{k})$ are written in terms of the polar and azimuthal angles, $\theta$ and $\phi$, as

$$
\begin{gathered}
\chi_{+}(\hat{k})=e^{i \phi / 2}\left(\begin{array}{c}
\cos \frac{\theta}{2} e^{-i \phi / 2} \\
\sin \frac{\theta}{2} e^{i \phi / 2}
\end{array}\right), \\
\chi_{-}(\hat{k})=e^{-i \phi / 2}\left(\begin{array}{c}
-\sin \frac{\theta}{2} e^{-i \phi / 2} \\
\cos \frac{\theta}{2} e^{i \phi / 2}
\end{array}\right),
\end{gathered}
$$

being mutually orthonormal, i.e., $\chi_{a}^{\dagger}(\hat{k}) \chi_{b}(\hat{k})=\delta_{a, b}$, with $a, b= \pm$.

\section{APPENDIX B: SCALAR, VECTOR, AND TENSOR CURRENTS IN THE JACOB-WICK CONVENTION}

Appendix B contains the list of the expressions for several scalar, vector and tensor currents in the kinematic configuration depicted in Fig. 1 to be used in the main text. For explicit current calculations, the chiral representation is adopted for four anticommutating Dirac gamma matrices $\gamma^{\mu}$ with $\mu=0,1,2,3$ and $\gamma_{5}=l \gamma^{0} \gamma^{1} \gamma^{2} \gamma^{3}$, whose expressions are given by the following $4 \times 4$ matrices

$$
\gamma^{\mu}=\left(\begin{array}{cc}
0 & \sigma_{+}^{\mu} \\
\sigma_{-}^{\mu} & 0
\end{array}\right) \quad \text { and } \quad \gamma_{5}=\left(\begin{array}{cc}
-1 & 0 \\
0 & 1
\end{array}\right)
$$

with $\sigma_{ \pm}^{\mu}=(1, \pm \vec{\sigma})$ in terms of three Pauli matrices $\vec{\sigma}=$ $\left(\sigma_{1}, \sigma_{2}, \sigma_{3}\right)$ defined by

$\sigma_{1}=\left(\begin{array}{ll}0 & 1 \\ 1 & 0\end{array}\right), \quad \sigma_{2}=\left(\begin{array}{cc}0 & -\imath \\ l & 0\end{array}\right), \quad \sigma_{3}=\left(\begin{array}{cc}1 & 0 \\ 0 & -1\end{array}\right)$.

The metric tensor is defined to be $g^{\mu \nu}=g_{\mu \nu}=\operatorname{diag}(1,-1$, $-1,-1)$.

First, the helicity-dependent scalar and pseudoscalar currents, surviving only when two helicities are same, i.e., $\lambda_{1}=\lambda_{2}$, read

$$
\begin{gathered}
u_{\lambda_{1} \lambda_{2}}^{+} \equiv \bar{u}_{1}\left(k_{1}, \lambda_{1}\right) v_{2}\left(k_{2}, \lambda_{2}\right) / m=\delta_{\lambda_{1}, \lambda_{2}}, \\
u_{\lambda_{1} \lambda_{2}}^{+} \equiv \bar{u}_{1}\left(k_{1}, \lambda_{1}\right) \gamma_{5} v_{2}\left(k_{2}, \lambda_{2}\right) / m=-\lambda_{1} \delta_{\lambda_{1}, \lambda_{2}},
\end{gathered}
$$

where $\lambda_{1,2}= \pm 1= \pm$ which is two times the helicity, $\pm 1 / 2$. Second, the helicity-dependent vector and axialvector currents, surviving only when two helicities are opposite, i.e., $\lambda_{1}=-\lambda_{2}$, read

$$
\begin{aligned}
& \bar{u}_{1}\left(k_{1}, \lambda_{1}\right) \gamma^{\mu} v_{2}\left(k_{2}, \lambda_{2}\right)=-\sqrt{2} m \delta_{\lambda_{1},-\lambda_{2}} \epsilon_{1}^{* \mu}\left(k_{1}, \lambda_{1}\right), \\
& \bar{u}_{1}\left(k_{1}, \lambda_{1}\right) \gamma^{\mu} \gamma_{5} v_{2}\left(k_{2}, \lambda_{2}\right)=-\sqrt{2} m \lambda_{1} \delta_{\lambda_{1},-\lambda_{2}} \epsilon_{1}^{* \mu}\left(k_{1}, \lambda_{1}\right),
\end{aligned}
$$

with $\lambda_{1,2}= \pm 1= \pm$ where the expression of the wave vector $\epsilon_{1}^{\mu}\left(k_{1}, \lambda_{1}\right)$ is given in Eq. (A10).

The contraction of the $X$ wave vector $\epsilon(p, \sigma)$ with $\sigma= \pm, 0$ and $k$ gives

$$
\begin{gathered}
k \cdot \epsilon(p, 0)=-m \cos \theta, \\
k \cdot \epsilon(p, \pm)= \pm m \frac{1}{\sqrt{2}} \sin \theta e^{ \pm i \phi},
\end{gathered}
$$

in terms of the polar and azimuthal angles, $\theta$ and $\phi$, in the $X \mathrm{RF}$.

For the sake of efficiently calculating and deriving the (reduced) helicity amplitudes of the decay $X \rightarrow M M$, we introduce four helicity-dependent quantities, $s_{\lambda_{1} \lambda_{2}}^{ \pm}$and $t_{\lambda_{1} \lambda_{2}}^{ \pm}$, defined by contracting the scalar and tensor operators given in Eqs. (18), (19), (20), and (21) with the $X$ and $M$ wave vectors appropriately as

$$
\begin{gathered}
s_{\lambda_{1} \lambda_{2}}^{ \pm}=\epsilon_{1}^{* \alpha}\left(k_{1}, \lambda_{1}\right) \epsilon_{2}^{* \beta}\left(k_{2}, \lambda_{2}\right) S_{\alpha \beta}^{ \pm}, \\
t_{\lambda_{1} \lambda_{2}}^{ \pm}=\epsilon_{1}^{* \alpha}\left(k_{1}, \lambda_{1}\right) \epsilon_{2}^{* \beta}\left(k_{2}, \lambda_{2}\right) T_{\alpha \beta, \mu \nu}^{ \pm} \epsilon^{\mu}(p,+) \epsilon^{\nu}(p,+),
\end{gathered}
$$

and two additional helicity-dependent quantities obtained by contracting the vector and axial-vector currents with the $X$ wave vector $\epsilon(p,+)$ as

$$
\begin{gathered}
v_{\lambda_{1} \lambda_{2}}^{+}=\bar{u}_{1}\left(k_{1}, \lambda_{1}\right) \gamma_{\mu} v_{2}\left(k_{2}, \lambda_{2}\right) \epsilon^{\mu}(p,+) / m, \\
v_{\lambda_{1} \lambda_{2}}^{-}=\bar{u}_{1}\left(k_{1}, \lambda_{1}\right) \gamma_{\mu} \gamma_{5} v_{2}\left(k_{2}, \lambda_{2}\right) \epsilon^{\mu}(p,+) / m,
\end{gathered}
$$

with $\lambda_{1,2}= \pm 1= \pm$. Explicitly the same-helicity quantities and the opposite-helicity quantities read

$$
\begin{gathered}
s_{ \pm \pm}^{+}= \pm s_{ \pm \pm}^{-}=1, \\
t_{+-}^{+}=(1+\cos \theta)^{2} / 2=2 d_{2,2}^{2}(\theta), \\
t_{-+}^{+}=(1-\cos \theta)^{2} e^{4 l \phi} / 2=2 d_{2,-2}^{2}(\theta) e^{4 l \phi}, \\
t_{ \pm \mp}^{-}= \pm t_{ \pm \mp}^{+},
\end{gathered}
$$

and the quantities involving the spinors in the fermionic case

$$
\begin{gathered}
u_{ \pm \pm}^{+}=\mp u_{ \pm \pm}^{-}=1, \\
v_{+-}^{+}=(1+\cos \theta) / \sqrt{2}=\sqrt{2} d_{1,1}^{1}(\theta), \\
v_{-+}^{+}=(1-\cos \theta) e^{2 l \phi} / \sqrt{2}=\sqrt{2} d_{1,-1}^{1}(\theta) e^{2 l \phi}, \\
v_{ \pm \mp}^{-}= \pm v_{ \pm \mp}^{+},
\end{gathered}
$$


in terms of the polar and azimuthal angles, $\theta$ and $\phi$, in the $X \mathrm{RF}$. All the quantities with other helicity combinations are zero.

The validity of the replacements in Eq. (33) can be confirmed with the four relations

$$
\begin{aligned}
& s_{ \pm \pm}^{+} u_{ \pm \pm}^{ \pm}=-s_{ \pm \pm}^{-} u_{ \pm \pm}^{\mp}, \\
& t_{ \pm \mp}^{+} v_{ \pm \mp}^{ \pm}=+t_{ \pm \mp}^{-} v_{ \pm \mp}^{\mp},
\end{aligned}
$$

which can be checked by use of all the expressions from Eq. (B13) to Eq. (B20). Moreover, in the general and covariant form, we have the corresponding four identities for nonzero decay helicity amplitudes

$$
\begin{aligned}
& S_{\alpha \beta}^{-} \epsilon_{1}^{* \alpha}\left(k_{1}, \pm\right) \epsilon_{2}^{* \beta}\left(k_{2}, \pm\right) \bar{u}_{1}\left(k_{1}, \pm\right)\left[1, \gamma_{5}\right] v_{2}\left(k_{2}, \pm\right) \\
& \quad=-S_{\alpha \beta}^{+} \epsilon_{1}^{* \alpha}\left(k_{1}, \pm\right) \epsilon_{2}^{* \beta}\left(k_{2}, \pm\right) \bar{u}_{1}\left(k_{1}, \pm\right)\left[\gamma_{5}, 1\right] v_{2}\left(k_{2}, \pm\right),
\end{aligned}
$$

$$
\begin{aligned}
& T_{\alpha \beta, \mu_{2} \mu_{3}}^{-} \epsilon_{1}^{* \alpha}\left(k_{1}, \pm\right) \epsilon_{2}^{* \beta}\left(k_{2}, \mp\right) \bar{u}_{1}\left(k_{1}, \pm\right) \gamma_{\mu_{1}}\left[1, \gamma_{5}\right] v_{2}\left(k_{2}, \mp\right) \\
& =T_{\alpha \beta, \mu_{2} \mu_{3}}^{+} \epsilon_{1}^{* \alpha}\left(k_{1}, \pm\right) \epsilon_{2}^{* \beta}\left(k_{2}, \mp\right) \bar{u}_{1}\left(k_{1}, \pm\right) \gamma_{\mu_{1}}\left[\gamma_{5}, 1\right] \\
& \quad \times v_{2}\left(k_{2}, \mp\right)
\end{aligned}
$$

where the last term $-g_{\perp \alpha \beta} k_{\mu_{2}} k_{\mu_{3}} / m^{2}$ of the tensor $T_{\alpha \beta, \mu_{2} \mu_{3}}^{+}$ defined in Eq. (20) does not contribute to the expression on the right-hand side of Eq.(B24) surviving only in the opposite-helicity case with $\lambda_{1}=-\lambda_{2}= \pm$.

The angle-dependent parts are encoded solely in the Wigner- $d$ functions and the reduced helicity amplitudes are independent of the helicity of the decaying particle. Therefore, it is sufficient to consider the cases with the maximal $X$ helicity $\sigma=J$ and the zero and two maximal helicity differences, $\lambda_{1}-\lambda_{2}=0, \pm 2 s$ for deriving the reduced helicity amplitudes. For them, three relevant Wigner- $d$ functions read

$$
\begin{aligned}
& d_{J, 0}^{J}(\theta)=\frac{(-1)^{J}}{2^{J}} \frac{\sqrt{(2 J) !}}{J !} \sin ^{J} \theta, \\
d_{J, 2 s}^{J}(\theta)= & \frac{(-1)^{J-2 s}}{2^{J}} \sqrt{\frac{(2 J) !}{(J+2 s) !(J-2 s) !}} \\
& \times(1+\cos \theta)^{2 s} \sin ^{J-2 s} \theta, \\
d_{J,-2 s}^{J}(\theta)= & \frac{(-1)^{J+2 s}}{2^{J}} \sqrt{\frac{(2 J) !}{(J+2 s) !(J-2 s) !}} \\
& \times(1-\cos \theta)^{2 s} \sin ^{J-2 s} \theta,
\end{aligned}
$$

which are to be factored out for deriving the reduced helicity amplitudes in Sec. IV.
[1] S. L. Glashow, Partial symmetries of weak interactions, Nucl. Phys. 22, 579 (1961).

[2] S. Weinberg, A Model of Leptons, Phys. Rev. Lett. 19, 1264 (1967).

[3] A. Salam, Weak and electromagnetic interactions, Conf. Proc. C 680519, 367 (1968).

[4] H. Fritzsch, M. Gell-Mann, and H. Leutwyler, Advantages of the color octet gluon picture, Phys. Lett. 47B, 365 (1973).

[5] B. P. Abbott et al. (LIGO Scientific and Virgo Collaborations), Observation of Gravitational Waves from a Binary Black Hole Merger, Phys. Rev. Lett. 116, 061102 (2016).

[6] B. P. Abbott et al. (LIGO Scientific and Virgo Collaborations), GW170817: Observation of Gravitational Waves from a Binary Neutron Star Inspiral, Phys. Rev. Lett. 119, 161101 (2017).

[7] B. P. Abbott et al. (LIGO Scientific and Virgo Collaborations), GWTC-1: A Gravitational-Wave Transient Catalog of Compact Binary Mergers Observed by LIGO and Virgo during the First and Second Observing Runs, Phys. Rev. X 9, 031040 (2019).

[8] P. A. Zyla et al. (Particle Data Group), Review of particle physics, Prog. Theor. Exp. Phys. 2020, 083C01 (2020).
[9] S. Weinberg and E. Witten, Limits on massless particles, Phys. Lett. 96B, 59 (1980).

[10] F. Loebbert, The Weinberg-Witten theorem on massless particles: An essay, Ann. Phys. (Berlin) 17, 803 (2008).

[11] X. Bekaert, N. Boulanger, and P. Sundell, How higher-spin gravity surpasses the spin two barrier: No-go theorems versus yes-go examples, Rev. Mod. Phys. 84, 987 (2012).

[12] L. D. Landau, On the angular momentum of a system of two photons, Dokl. Akad. Nauk SSSR 60, 207 (1948).

[13] C. N. Yang, Selection rules for the dematerialization of a particle into two photons, Phys. Rev. 77, 242 (1950).

[14] G. Aad et al. (ATLAS Collaboration), Observation of a new particle in the search for the Standard Model Higgs boson with the ATLAS detector at the LHC, Phys. Lett. B 716, 1 (2012).

[15] S. Chatrchyan et al. (CMS Collaboration), Observation of a new boson at a mass of $125 \mathrm{GeV}$ with the CMS Experiment at the LHC, Phys. Lett. B 716, 30 (2012).

[16] S. Y. Choi, M. M. Muhlleitner, and P. M. Zerwas, Theoretical basis of Higgs-spin analysis in $H \rightarrow \gamma \gamma$ and $Z \gamma$ decays, Phys. Lett. B 718, 1031 (2013).

[17] V. Pleitez, The angular momentum of two massless fields revisited, arXiv:1508.01394. 
[18] E. Leader, Spin in particle physics, Cambridge Monogr. Part. Phys., Nucl. Phys., Cosmol. 15, 78 (2011).

[19] A. P. Balachandran, S. G. Jo, and G. Marmo, in Group Theory and Hopf Algebra: Lectures for Physicists (World Scientific Publishing, Singapore, 2010), see pp. 191 and 192.

[20] A. P. Balachandran and S. G. Jo, $Z^{0} \rightarrow 2 \gamma$ and the twisted coproduct of the Poincare group, Int. J. Mod. Phys. A 22, 6133 (2007).

[21] W. Beenakker, R. Kleiss, and G. Lustermans, No LandauYang in QCD, arXiv:1508.07115.

[22] M. Cacciari, L. Del Debbio, J. R. Espinosa, A. D. Polosa, and M. Testa, A note on the fate of the Landau-Yang theorem in non-Abelian gauge theories, Phys. Lett. B 753, 476 (2016).

[23] V. Pleitez, Angular momentum and parity of a two gluon system, arXiv:1801.09294.

[24] W. Behr, N. G. Deshpande, G. Duplancic, P. Schupp, J. Trampetic, and J. Wess, The $Z \rightarrow \gamma \gamma, g g$ decays in the noncommutative standard model, Eur. Phys. J. C 29, 441 (2003).

[25] S. Weinberg, Causality, anti-particles and the spin statistics connection in higher dimensions, Phys. Lett. 143B, 97 (1984).

[26] I. P. Ivanov, V. G. Serbo, and P. Zhang, Fate of the LandauYang theorem for twisted photons, J. Opt. 21, 114001 (2019).

[27] M. Jacob and G. C. Wick, On the general theory of collisions for particles with spin, Ann. Phys. (N.Y.) 7, 404 (1959).
[28] N. Arkani-Hamed, T. C. Huang, and Y.t. Huang, Scattering amplitudes for all masses and spins, arXiv:1709.04891.

[29] H.E. Haber, Spin formalism and applications to new physics searches, arXiv:hep-ph/9405376.

[30] M. E. Rose, Elementary Theory of Angular Momentum (Dover Publication Inc., New York, 2011).

[31] R. E. Behrends and C. Fronsdal, Fermi decay of higher spin particles, Phys. Rev. 106, 345 (1957).

[32] P. R. Auvil and J. J. Brehm, Wave functions for particles of higher spin, Phys. Rev. 145, 1152 (1966).

[33] S. Weinberg, The Quantum Theory of Fields. Vol. 1: Foundations (Cambridge University Press, Cambridge, England, 1995).

[34] S. Y. Choi and J. H. Jeong, Vector currents of integer-spin Majorana particles, arXiv:2102.01317.

[35] A. Denner, H. Eck, O. Hahn, and J. Kublbeck, Feynman rules for fermion number violating interactions, Nucl. Phys. B387, 467 (1992).

[36] A. Denner, H. Eck, O. Hahn, and J. Kublbeck, Compact Feynman rules for Majorana fermions, Phys. Lett. B 291, 278 (1992).

[37] B. Kayser, Majorana neutrinos and their electromagnetic properties, Phys. Rev. D 26, 1662 (1982).

[38] B. Kayser, $C P T, C P$, and $C$ phases and their effects in Majorana particle processes, Phys. Rev. D 30, 1023 (1984).

[39] F. Boudjema and C. Hamzaoui, Massive and massless Majorana particles of arbitrary spin: Covariant gauge couplings and production properties, Phys. Rev. D 43, 3748 (1991). 